\title{
Satisfacción con la vida y sus correlatos socio-personales en adolescentes de secundarias públicos de Sonora, México
}

\author{
Angel Emigdio Lagarda Lagarda ${ }^{1}$, José Ángel Vera Noriega², Jesús Tánori \\ Quintana $^{3}$ \\ Instituto Tecnológico de Sonora-México y Centro de Investigación de \\ Alimentación y Desarrollo-México
}

El objetivo de este trabajo fue medir la relación de las variables personales y de contexto, satisfacción con la vida y el bienestar personal de adolescentes escolarizados en el noroeste de México. El diseño del estudio es no experimental ex post facto de tipo transversal. Se formaron submuestras de bajos-altos niveles de satisfacción con la vida y bienestar personal. Se realizaron análisis discriminantes y regresiones logísticas. El índice de bienestar local, el autoconcepto familiar y el autoconcepto social resultan como variables de protección del bienestar personal, sumándose el apego al barrio en la predicción de la satisfacción con la vida. Se concluyó que es importante desarrollar modelos que incluyan variables multinivel para explicar y predecir el bienestar subjetivo.

Palabras clave: satisfacción con la vida, estudiantes de secundaria, autoconcepto, apego al barrio.

Satisfaction with life and its socio-personal correlates in adolescents from public middle schools in Sonora, Mexico

The objective of the study was to measure the relationship of personal variables and context with satisfaction with the life and personal well-being of school adolescents in northwestern Mexico. The design is non-experimental ex post facto of transversal type. Two samples of high-low satisfaction with life and personal well-being were compared. Discriminant analyzes and logistic regressions were performed. In the analyzes, the Local Well-being Index, the Family Self-concept and the Social Self-concept result as predictive variables of Personal Well-being, adding to the Neighborhood Attachment in the prediction of Satisfac-

1 Estudiante de Doctorado en Innovación Educativa por la Universidad de Sonora. Dirección postal: Edificio 9B Segundo piso, Blvd. Luis Encinas y Rosales s/n, Col. Centro, C.P. 83000. Contacto: angelagarda@gmail.com https://orcid.org/0000-0003-1078-4243

2 Doctor en Psicología Social. Profesor-investigador en el Centro de Investigación de Alimentación y Desarrollo. Dirección Postal: Centro de Investigación en Alimentación y Desarrollo, A.C. Carretera a la Victoria Km. 0.6 Hermosillo, Sonora, México. Apartado Postal: 83000. Contacto: avera@ciad.mx https://orcid.org/0000-0003-2764-4431

3 Doctor en Ciencias Sociales. Profesor-investigador en el Instituto Tecnológico de Sonora, México. Dirección Postal: Instituto Tecnológico de Sonora 5 de Febrero 818 Sur, Col. Centro, Ciudad Obregón, Sonora, México. Contacto: jesus.tanori@iston.edu.mx https://orcid.org/ 0000-0002-6485-2267 
tion with Life. It is concluded that it is important to develop ecological models that include multilevel variables to explain and predict the subjective well-being.

Keywords: life satisfaction, middle school students, self-concept, neighborhood attachment.

Satisfaçáo com a vida e seus correlatos sócio-pessoais em adolescentes de escolas públicas de ensino médio em Sonora, México

O objetivo foi verificar a relação das variáveis pessoais e de contexto com a satisfação com a vida e o bem estar pessoal dos adolescentes escolarizados do Noroeste do México. O delineamento do estudo é náo experimental ex post facto do tipo transversal. Foram duas sub-amostras baixos-altos níveis de satisfação com a vida e bem-estar pessoal. Foram realizadas análises discriminante e regressóes logísticas. De acordo com as análises o Bem Estar Local, o Autoconceito Familiar e o Autoconceito Social foram preditores do Bem Estar Pessoal, além do Apego ao Bairro na predição da Satisfação com a Vida. Conclui-se que é importante desenvolver modelos ecológicos que incluam variáveis multiníveis para explicar e predizer o bem-estar subjetivo.

Palavras-chave: satisfaçáo com a vida, 3. ${ }^{\circ}$ Ciclo do Ensino Básico, autoconceito, apego ao bairro.

Satisfaction à l'égard de la vie et de ses corrélats socio-personnels chez les adolescents des écoles secondaires publiques de Sonora, au Mexique.

L'objectif de ce travail était de mesurer la relation entre les variables personnelles et le contexte avec la satisfaction à l'égard de la vie et du bien-être personnel des adolescents scolarisés dans le nord-ouest du Mexique. La conception de l'étude est ex-facto non expérimentale de type transversal. Ils étaient divisés en hauts et en bas pour la satisfaction de la vie et le bienêtre personnel. Des analyses discriminantes et des régressions logistiques ont été effectuées. L'indice de bien-être local, le concept de famille et le concept de société en tant que variables prédictives du bien-être personnel, renforcent l'attachement du quartier à la prévision de la satisfaction de la vie. En conclusion, il est important de développer des modèles écologiques intégrant des variables multiniveaux pour expliquer et prédire le bien-être subjectif.

Mots-clés: satisfaction de la vie, premier cycle du secondaire, concept de soi, attachement au quartier. 
En los últimos años han aumentado las investigaciones que abordan el bienestar subjetivo originando que el estudio de este sea considerado como un objetivo de las políticas públicas de países desarrollados y en desarrollo. La evidencia de que los ingresos y el bienestar subjetivo solo están vinculados cuando existen niveles de ingresos muy bajos ha provocado un interés en el estudio de los aspectos no materiales del bienestar (PNUD, 2011).

En la actualidad el bienestar subjetivo es fundamental para estudiar la calidad de vida de una población. En 2013 la Organización para la Cooperación y el Desarrollo Económico recomendó que el bienestar subjetivo fuera objeto de análisis por parte de los organismos estadísticos nacionales de los países miembros a esta organización, reconociendo que se necesita recolectar información a través de muestras más grandes y representativas de diferentes grupos de población (OECD, 2013).

La dimensión más estudiada del bienestar subjetivo es la de satisfacción con la vida, esto se debe a que tiene más variación internacionalmente y es más sencilla su explicación con base en las circunstancias de vida (Helliwell et al, 2017).

En México las situaciones asociadas a los niveles más altos de bienestar subjetivo son el no presentar condiciones de pobreza y vulnerabilidad, el haber obtenido un aumento de sueldo, confiar en cinco o más amistades y la participación en redes sociales, mientras que en las situaciones asociadas a los niveles más bajos de bienestar subjetivo se encuentran la drogadicción en el hogar, el no confiar en ningún familiar, no tener amistades, el considerar que no se han tomado decisiones importantes a lo largo de la vida y que el nivel de vida actual es menor al de su niñez (INEGI, 2015).

En la población indígena se ha encontrado que están satisfechos con su vida en lo general, pero en dominios específicos referentes a su marginalidad y carencia social obtienen niveles bajos (González et al., 2015). 
Vera et al. (2017) encontraron que las personas con discapacidad auditiva, que además son de bajos recursos económicos, suelen presentar bajos niveles de satisfacción con la vida. En cuanto a personas con discapacidad motriz se encontró que presentan un nivel de satisfacción con la vida menor al de la media nacional (Vera, Tánori et al., 2018).

Sin embargo, la documentación existente da cuenta de los procesos de bienestar en personas en la edad de 18 a 60 años, por lo cual queda un vacío en poblaciones de infantes y jóvenes, ya que no se cuenta con una valoración como la de los adultos (Casas, 2011; Tánori et al., 2013) para analizar cómo la satisfacción con la vida de las personas se asocia con variables contextuales, particularmente en las diferencias existentes que hay por la pertenencia a la comunidad y la percepción de inseguridad (Alfaro et al., 2015; Benatuil; 2003).

Diferentes estudios en adultos han demostrado la influencia de factores culturales y sociales en la satisfacción con la vida. Sin embargo, en los pocos estudios realizados con la población joven, la satisfacción con la vida ha estado relacionada con el ambiente en el que se desarrolla, siendo las variables relación con los padres de familia y el apoyo social las que han jugado un rol importante (Huebner, 2004).

La calidad de vida, desde la una perspectiva subjetiva, ha sido objeto de estudio en diversas investigaciones realizadas a diferentes grupos de personas a través de variables tanto objetivas como subjetivas. Sin embargo, las variables subjetivas y su relación con el contexto comunitario y variables personales, no han sido analizadas lo suficiente y menos en la población adolescente (Casas et al., 2013; Arita, 2010; Palomar, 2010).

El bienestar subjetivo durante la adolescencia ha sido escasamente estudiado en comparación con el bienestar subjetivo en adultos (Huebner, 2004). Además, los trabajos realizados en México donde estudiaron el efecto de un ambiente comunitario violento o en vulnerabilidad con el bienestar de los jóvenes (Martínez-Ferrer et al., 2016).

La adolescencia es una etapa determinante donde el individuo, al mantener una sana relación con su contexto, puede lograr un sano 
desarrollo y desplegar todas sus capacidades, prosperando como persona prosocial y responsable (Oliva et al., 2011).

Sin embargo, son pocos los estudios sobre bienestar subjetivo con adolescentes latinoamericanos (Castellá et al., 2012). Además, los resultados de la escasa investigación existente difieren entre sí. En un estudio con adolescentes de 12 a 15 años de Brasil, Sudáfrica, India, España y Noruega, se observó una asociación negativa entre el bienestar subjetivo y la edad (Coenders et al., 2005), estos resultados concuerdan con los encontrados por Casas et al. (2009), pero se contraponen a los encontrados con poblaciones latinoamericanas universitarias, donde en una edad de 18 a 25 años no ha resultado ser una variable significativa (Vera et al., 2010; Vera y Rodríguez, 2007).

El bienestar subjetivo se conforma de dos partes: una afectiva y otra cognitiva. La parte afectiva se refiere al equilibrio entre emociones positivas, como la alegría, y emociones negativas, como la ansiedad, mientras que la parte cognitiva (llamada normalmente satisfacción con la vida) está relacionada a la satisfacción del sujeto con su vida en general o en distintos dominios (Diener, 1984; Cummins, 1997; Schmidt et al., 2014).

Cummins (2000) propone la satisfacción con la vida como el indicador principal de su teoría homeostática del bienestar subjetivo. Dicha teoría propone que el bienestar subjetivo funciona en un nivel abstracto y que se puede medir realizando una pregunta general a las personas sobre qué tan satisfechos están con sus vidas como un todo. Según Cummins (2000), a pesar de ser una pregunta muy general, la respuesta reflejaría el estado de bienestar subjetivo de las personas, el cual sería el producto del sistema homeostático como proceso de adaptación.

Dicho sistema tiene el objetivo de generar un sentido positivo de bienestar personalizado por parte del individuo que está haciendo la valoración. La estimación que se hace de la vida en general no funciona para conocer la satisfacción que se tiene sobre los diferentes componentes o dominios de la vida de las personas. Para conocer la satisfacción de estos dominios se debe preguntar específicamente por el nivel de satisfacción con ellos. Estas preguntas tienen como objetivo conocer aspectos de la vida donde es procesada información más espe- 
cífica, y con ello el control del sistema homeostático disminuye. Esto provoca que la satisfacción que se tiene por los diferentes dominios pueda ser mayor o menor al nivel general de satisfacción por la vida como un todo (Arita, 2014; Cummins et al. 2003).

Cummins et al. (2003) relacionan lo anterior con las dimensiones proximal-distal. De esta manera, la influencia homeostática disminuye a medida que se valora desde lo proximal, que en este caso sería el dominio personal a lo más distal: familia, amigos y sociedad. Por lo tanto, cuando se valora la satisfacción personal (proximal) el control homeostático tiene baja sensibilidad para esta evaluación y la variabilidad es menor, mientras que cuando se evalúa la sociedad (distal), la sensibilidad es mayor y en consecuencia puede existir mayor variabilidad debido a que la homeostasis tiene como objetivo principal sostener un sentido de bienestar personal.

Según Veenhoven (2008) las condiciones que fomentan el bienestar subjetivo son la modernidad en la que se vive actualmente, necesidades básicas cumplidas, la equidad social, la participación social y el apoyo social.

Diener (1994) menciona que el autoconcepto es uno de los mejores predictores sobre el bienestar subjetivo que existen. Casas et al. (2007) y Garaigordobil et al. (2009) encontraron una correlación significativa entre la satisfacción con la vida como un todo, así como de sus dominios con el autoconcepto, por lo que se ha sugerido estudiar las diferentes dimensiones del autoconcepto y su relación con los diferentes dominios de la satisfacción con la vida en los estudiantes. (Vera et al., 2002; Martínez et al., 2007)

El autoconcepto juega, por lo tanto, una parte fundamental en el entendimiento de la manera en que la personalidad promueve el bienestar en las personas (Vera et al., 2007). Entendida como la percepción que tiene una persona de sí misma, dicha percepción se forma a través de la experiencia con su entorno, especialmente por refuerzos ambientales, entre otros.

El constructo del autoconcepto es sumamente importante y útil para explicar y poder predecir cómo se comporta una persona. Se piensa 
que las percepciones que uno tiene de sí mismo influyen en la forma en que se actúa, y los actos a su vez influyen en la manera en que se percibe a sí mismo. El autoconcepto se deduce de las respuestas que una persona manifiesta ante las situaciones donde, dichas respuestas o situaciones pueden ser simbólicas o físicas (Shavelson et al. 1976).

Por otro lado, el contexto comunitario y sociocultural donde se desarrollan los individuos provee a las personas de un conjunto de características que influyen en sus comportamientos y actitudes (Tánori, 2012). La participación e integración de los adolescentes en su comunidad es un punto clave junto con la dimensión de la familia y la escuela para reducir la frecuencia de violencia y el fortalecimiento de redes de apoyo (Moreno-Ruiz et al., 2012). Además, uno de los mayores efectos de la inseguridad en la comunidad es el deterioro de las interacciones sociales (Martínez-Ferrer et al., 2016).

Oliva et al. (2011) destacan que la estimación que realizan los adolescentes sobre su propia colonia puede resultar de gran apoyo para explicar la relación que guarda el contexto comunitario y el desarrollo de los jóvenes que en él habitan. A partir de lo anterior es que se ha propuesto el concepto de activos para el desarrollo o apego al barrio, estos son los recursos personales, familiares, escolares o comunitarios que promueven la competencia y el desarrollo adolescente y, a su vez, previenen la aparición de problemas (Oliva et al., 2012).

Se ha demostrado que algunos activos de barrio, como el apego a vecindario y la seguridad se encuentran relacionados con el bienestar de los adolescentes que viven en él. Cuando el joven se integra en su comunidad tiende a percibir un mayor bienestar (Moreno-Ruiz et al., 2012). Estudios han constatado una disminución de la satisfacción con la vida en los ciudadanos que perciben su comunidad como un lugar inseguro (Cohen, 2008).

El objetivo del presente estudio fue analizar en qué medida los factores personales y de contexto afectan los niveles de bienestar personal y satisfacción con la vida de los estudiantes de las escuelas secundarias en Sonora. 


\section{Método}

\section{Participantes}

La población se conformó por estudiantes de secundarias públicas del estado de Sonora, México. Dichas escuelas se ubican geográficamente en zonas identificadas como polígonos de violencia intrafamiliar -OCCSES (2016)-. La muestra consistió en 1212 estudiantes de diez escuelas secundarias, de los cuales 661 son mujeres y 551 hombres. El $41.2 \%$ de los estudiantes cursaban el primer grado y un $29.2 \%$ y $29.4 \%$ el segundo y tercer grado correspondientemente: 854 pertenecientes al turno matutino y 358 al vespertino. La media de la edad fue de 13.1 años $(D E=.95)$. El 9.6\% de los jóvenes cuentan con un empleo remunerado. 78 jóvenes mencionan ser golpeados por alguno de sus padres y un $25 \%$ reporta tener al menos un amigo perteneciente a una pandilla.

\section{Medición}

Para todas las escalas se realizó el mismo procedimiento de validación. Dicho proceso consistió en un análisis factorial confirmatorio (AFC), ya que se contaba con teorías y estructuras de cada medida, por lo tanto, se confirmó si se ajustaban a la población del presente estudio.

Se utilizó la adaptación y validación de Galindez y Casas (2011) de la Escala multidimensional de satisfacción con la vida para estudiantes (MSLSS por sus siglas en inglés) de Huebner (2001), que pretende evaluar diferentes aspectos de la vida de los jóvenes. Dicha escala se conformó de 40 reactivos en escala Likert de 5 puntos, donde 0 se refiere a un completo desacuerdo y 4 un completo acuerdo. Los ítems se organizaron en cinco dimensiones: 1) la dimensión familia, y se conformó por siete reactivos (ej. Los miembros de mi familia se la llevan bien juntos); 2) la dimensión amigos, se conformó por nueve reactivos (ej. Mis amigos son grandiosos); 3) la dimensión escuela, se compuso por ocho reactivos (ej. Me gusta estar en la escuela); 4) la dimensión satisfacción con la colonia, nueve reactivos (ej. Me gusta mi colonia) y; 
5) la dimensión personal, que se compuso de siete reactivos (ej. Soy una persona amable). Se llevó a cabo el AFC con la estructura original de la escala conformada por 40 reactivos distribuidos en cinco dimensiones. En los análisis se tuvieron que eliminar 18 reactivos que estaban imposibilitando que el modelo ajustara, de esta manera la escala quedó conformada por 22 ítems, distribuidos en las cinco dimensiones originales. Todos los indicadores de ajuste al modelo resultaron satisfactorios $(\mathrm{CMIN} / \mathrm{DF}=3.287 ; \mathrm{GFI}=.952 ; \mathrm{CFI}=.954 ; \mathrm{RMSEA}=.043 ; \mathrm{SRMR}=$ $.034)$.

Índice de Bienestar Personal (PWI) (Cummins, et al., 2003). en escala tipo Likert de cinco niveles, los cuales conformaron un continuo de "totalmente insatisfecho" a "totalmente satisfecho" y se compuso por nueve ítems que indagaron sobre la situación económica, salud, logros, relaciones personales, su colonia y seguridad en la vida, todas a un nivel personal (ej.Tu salud). En la validación del PWI no fue necesario eliminar ningún ítem, quedando conformado por los nueve reactivos originales mostrando un buen ajuste al modelo $(\mathrm{CMIN} / \mathrm{DF}=2.345$; $\mathrm{GFI}=.981 ; \mathrm{CFI}=.984 ; \mathrm{RMSEA}=.043 ; \mathrm{SRMR}=.025)$.

Índice de bienestar local (IBL) (Cummins, et al., 2003): escala tipo Likert de cinco niveles, cuyas opciones de respuesta fueron de "totalmente insatisfecho" a "totalmente satisfecho". Se conformó de ocho reactivos que cuestionan la satisfacción de los jóvenes con la ciudad donde viven (ej. La vida en tu ciudad). Después de eliminar tres ítems de la escala debido a los índices de modificación todos los indicadores de ajuste del modelo fueron excelentes $(C M I N / D F=2.265 ; G F I=.991$; $C F I=.991 ; R M S E A=.042 ; S R M R=.022$ ).

Escala para la evaluación de los activos del barrio, (Oliva, et al., 2011): dicha escala se conformó por 22 reactivos tipo Likert de 5 niveles, cuyas respuestas fueron de "totalmente falso" a "totalmente verdadero". La escala evaluó la percepción de los y las adolescentes sobre diversos factores de la colonia donde habitan. La estructura factorial fue de cinco factores: 1) apoyo y empoderamiento de la juventud, que se conformó de seis reactivos (ej. La gente adulta de mi colonia valora mucho a los jóvenes); 2) apego al barrio, conformada por cuatro ítems 
(ej. Me siento muy unido a mi colonia.); 3) seguridad del barrio, contiene cuatro reactivos (ej. La gente de mi colonia comete delitos); 4) control social, cuatro ítems (ej. En mi colonia, si haces cualquier daño seguro que algún adulto te regañará) y; 5) actividades para jóvenes compuesta también por cuatro reactivos (ej. Hay pocas colonias en las que haya tantas actividades para jóvenes como en el mía). La dimensión seguridad del barrio era negativa, por lo que se procedió a recodificarla para contar con la misma orientación que las demás dimensiones. El AFC se conformó igualmente por cinco dimensiones, mostrando a la dimensión de seguridad con covarianzas muy bajas con las cuatro dimensiones restantes. Sin embargo, el modelo presenta indicadores de ajuste adecuados $(\mathrm{CMIN} / \mathrm{DF}=3.206 ; \mathrm{GFI}=.951 ; \mathrm{CFI}=.945 ; \mathrm{RMSEA}=.043$; SRMR $=.038)$.

Se utilizó la Escala de autoconcepto escolar de Malo et al. (2011), la cual fue una adaptación de la escala AF5 de García y Musitsu (1999). Dicho instrumento se conformó de 24 reactivos que se distribuyen en cuatro dimensiones: autoconcepto académico (ej. Soy un buen estudiante), autoconcepto familiar (ej. Mis padres confian en mí), autoconcepto físico (ej. Soy una persona atractiva) y autoconcepto social (ej. Mis amigos/as me aprecian), resultando de seis reactivos cada una. La escala, de respuesta, fue de tipo Likert con cinco opciones de respuesta, las cuales establecieron un continuo de "nunca" a "siempre". En el proceso de validación de la medida de autoconcepto conservó sus cuatro dimensiones con un total de 16 reactivos de los 24 iniciales. La covarianza de menor puntaje fue de 0.54 entre las dimensiones física y académica, mientras que la mayor fue de 0.71 entre la dimensión física y social. Los indicadores de ajuste al modelo del AFC (CMIN/ $D F=3.889 ; G F I=.962 ; C F I=.956 ; R M S E A=.049 ; S R M R=.037)$.

\section{Procedimiento}

Se acudió a los planteles, se contó con el apoyo de directores, prefectos y psicólogos de las escuelas secundarias y fueron seleccionados aleatoriamente dos grupos de primer grado, un grupo de segundo y un grupo más de tercero. Un miembro capacitado del grupo de inves- 
tigación aplicó en cada grupo la entrega de los cuadernillos, hojas de respuesta electrónica explicando las instrucciones para el correcto llenado de las formas, así como mencionando el carácter voluntario y confidencial de su participación. Una vez reunidos los datos la captura se realizó con lector óptico para después proceder a la depuración, codificación y sustitución de los datos perdidos. Posteriormente se realizaron los análisis estadísticos para cumplir con los objetivos del estudio, los cuales consistieron en la prueba de la función discriminante y regresión logística con el programa estadístico SPSS 22.

\section{Análisis de datos}

Una vez que se construyó y depuró la base, con el objetivo de conocer las variables de riesgo y protección, se formaron dos variables dummy con base a la media teórica del PWI y la MSLSS. De esta manera los valores que estuvieron por debajo de una puntuación de 2 se recodificaron con valor de 0 , mientras que los que estuvieron por arriba, se recodificaron con valor de 1 . Estas funcionaron para realizar los análisis multivariantes necesarios, los cuales fueron el análisis discriminante y la regresión logística. Dichos análisis se realizaron con el método de selección por pasos hacia adelante. Se tomaron los valores mínimos de .75 como porcentaje de correcta clasificación (de los grupos); .50 para la R de Nagelkerke y un valor de significancia de .05 para Wald. Se examinaron las variables predictoras analizando sus coeficientes beta ( $\beta$ ) para determinar si explican significativamente y convirtiendo los exponenciales a porcentajes utilizando la fórmula “(ExpB-1) x 100".

\section{Resultados}

De manera inicial, como resultados, se presentan los análisis descriptivos, tanto de las variables y sus dimensiones que se aplicaron en el estudio. En la tabla 1 se presentan las medias y desviación estándar. Se puede interpretar que la mayoría de los jóvenes estudiantes tienden estar de acuerdo con las afirmaciones y situaciones que se les presentan, 
en relación con la satisfacción con la vida de los escolares, su bienestar personal, bienestar por la ciudad donde radican, los activos en su barrio y su autoconcepto en un sentido positivo. La dimensión con el puntaje medio más bajo es la seguridad del barrio donde vive. Además, los valores de sesgo y curtosis sugieren que la distribución de las respuestas en los reactivos tiende a la normalidad univariada, mediante el cumplimiento del rango de \pm 1.5 en asimetría y curtosis propuesto por George y Mallery (2003), por lo que se posibilitan análisis paramétricos sin algún problema de sesgo. Finalmente, se observa que los valores mínimos y máximos son los establecidos, de manera que van de cero a cuatro, recorriendo todo el espectro de respuesta, de cinco puntos.

\section{Tabla 1}

Análisis descriptivo de las variables y sus dimensiones.

\begin{tabular}{lcccccc}
\hline Variables y Dimensiones & $M$ & $D E$ & Asimetría & Curtosis & Mínimo & Máximo \\
\hline Bienestar personal & 2.86 & 0.82 & -.82 & .21 & 0 & 4 \\
Bienestar local & 2.11 & 0.92 & -.05 & -.47 & 0 & 4 \\
Satisfacción con amigos & 2.97 & 0.92 & -.78 & -.02 & 0 & 4 \\
Satisfacción personal & 2.85 & 0.85 & -.67 & .09 & 0 & 4 \\
Satisfacción con la escuela & 2.86 & 0.95 & -.60 & -.28 & 0 & 4 \\
Satisfacción con la familia & 3.04 & 0.88 & -.96 & .29 & 0 & 4 \\
Satisfacción con el ambiente & 2.62 & 1.04 & -.51 & -.60 & 0 & 4 \\
Apoyo y empoderamiento de & 2.12 & 1.01 & -.07 & -.54 & 0 & 4 \\
la juventud & & & & & & 4 \\
Apego al barrio & 2.36 & 0.94 & -.23 & -.38 & 0 & 4 \\
Seguridad del barrio & 1.94 & 1.00 & -.05 & -.62 & 0 & 4 \\
Control social & 2.55 & 0.93 & -.40 & -.28 & 0 & 4 \\
Actividades para jóvenes & 2.03 & 0.93 & -.10 & -.31 & 0 & 4 \\
Autoconcepto académico & 2.55 & 0.80 & -.18 & -.30 & 0 & 4 \\
Autoconcepto familiar & 3.02 & 0.85 & -1.07 & .72 & 0 & 4 \\
Autoconcepto físico & 2.43 & 0.83 & -.20 & -.47 & 0 & 4 \\
Autoconcepto Social & 2.78 & 0.86 & -.58 & -.07 & 0 & 4 \\
\hline
\end{tabular}




\section{Análisis discriminante del PWI}

Al realizar el análisis discriminante del PWI, se llevaron a cabo tres pasos, resultando tres variables discriminantes. En el primer paso resultó el IBL, en el segundo paso se incluyó el autoconcepto familiar, y por último, en el tercer paso, se incluyó el autoconcepto social. Los valores de Lambda de Wilks se consideran aceptables ya que van desde .601, el más bajo para el autoconcepto social, hasta .803 para el IBL, siendo valores cercanos a 1 los que tienen mayor capacidad de discriminación entre grupos (ver tabla 2).

\section{Tabla 2}

Variable predictoras en un análisis discriminante por pasos para el IBP.

\begin{tabular}{clcccc}
\hline Paso & Variable predictora & $\begin{array}{c}\text { Variables en la función } \\
\text { discriminante }\end{array}$ & $\begin{array}{c}\lambda \text { de } \\
\text { Wilks }\end{array}$ & $F$ & $p$ \\
\hline 1 & Índice de bienestar local & 1 & .803 & 127.54 & .000 \\
2 & Autoconcepto familiar & 2 & .629 & 21.60 & .000 \\
3 & Autoconcepto social & 3 & .601 & 5.12 & .000 \\
\hline
\end{tabular}

Los valores anteriores son respaldados con los coeficientes de función discriminante canónica estandarizadas y la matriz con los coeficientes de estructura, los cuales hicieron posible conocer cuáles son las variables que más influyen en el modelo de predicción. Así, el IBL obtuvo los coeficientes .806 y .844, que son los más cercanos a 1 y por lo tanto se puede considerar como la variable más relevante en el modelo de discriminación (tabla 3). 


\section{Tabla 3}

Correlación de variables de predicción con funciones discriminantes $y$ coeficientes estandarizados de la función discriminante.

\begin{tabular}{lcc}
\hline \multicolumn{1}{c}{ Variable predictora } & $\begin{array}{c}\text { Correlación con } \\
\text { función discriminante }\end{array}$ & $\begin{array}{c}\text { Coeficientes de función } \\
\text { discriminante estandarizados }\end{array}$ \\
\cline { 2 - 3 } & Función 1 & Función1 \\
\hline Índice de bienestar local & .806 & .844 \\
Autoconcepto familiar & .414 & .536 \\
Autoconcepto social & .207 & .471 \\
\hline
\end{tabular}

$\mathrm{El}$ análisis discriminante permitió clasificar al 78.8\% de la población global. El modelo clasificó en mejor medida al grupo de los adolescentes sin bienestar personal con un $81.3 \%$, mientras que a los que reportan bienestar personal los clasificaron en un $76.4 \%$.

\section{Análisis discriminante de la MSLSS}

En el análisis discriminante de la MSLSS se efectuaron cuatro pasos y resultaron cuatro variables como discriminantes. En el primer paso resultó el autoconcepto familiar, en el segundo paso se incluyó el autoconcepto social, en el tercer paso se sumó el IBL, y finalmente en el cuarto paso se agregó el apego al barrio. Los valores de Lambda de Wilks fueron aceptables puesto que el valor mínimo fue de 0.604 (tabla 4).

Los coeficientes de función discriminante canónica estandarizada y la matriz con los coeficientes de estructura apoyan lo anterior. El autoconcepto familiar y el autoconcepto social obtuvieron los coeficientes más cercanos a uno siendo las variables predictoras más importantes del modelo (tabla 5). 


\section{Tabla 4}

Variables de predicción en un análisis discriminante por pasos para la MSLSS.

\begin{tabular}{clcccc}
\hline Paso & Variable predictora & $\begin{array}{c}\text { Variables en la función } \\
\text { discriminante }\end{array}$ & $\begin{array}{c}\lambda \text { de } \\
\text { Wilks }\end{array}$ & $F$ & $p$ \\
\hline 1 & Autoconcepto familiar & 1 & .651 & 124.35 & .000 \\
2 & Autoconcepto social & 2 & .630 & 87.36 & .000 \\
3 & Índice de bienestar local & 3 & .605 & 67.67 & .000 \\
4 & Apego al barrio & 4 & .604 & 54.40 & .000 \\
\hline
\end{tabular}

\section{Tabla 5}

Correlación de variables de predicción con funciones discriminantes $y$ coeficientes estandarizados de la función discriminante.

\begin{tabular}{lcc}
\hline \multicolumn{1}{c}{ Variable predictora } & $\begin{array}{c}\text { Correlación con } \\
\text { función discriminante }\end{array}$ & $\begin{array}{c}\text { Coeficientes de función } \\
\text { discriminante estandarizados }\end{array}$ \\
\cline { 2 - 3 } & Función 1 & Función1 \\
\hline Autoconcepto familiar & .521 & .752 \\
Autoconcepto social & .435 & .669 \\
Índice de bienestar local & .291 & .458 \\
Apego al barrio & .292 & .630 \\
\hline
\end{tabular}

Para finalizar los resultados del análisis discriminante de la MSLSS, el modelo clasificó al grupo de los insatisfechos en un 80.9\%, mientras que a los satisfechos en un $80.3 \%$, por lo cual se puede decir, que, aunque por poco, clasificó mejor al grupo de los insatisfechos. Las cuatro variables permitieron agrupar al 80.6\% de los sujetos.

\section{Análisis de regresión logística para el PWI}

Primero se realizó la regresión logística con el PWI como variable dependiente, ahí se obtuvo que el valor de $\mathrm{r}^{2}$ de Nagelkerke fue de .53, esto representa que las variables protectoras forman un modelo con una 
capacidad predictora adecuada. En cuanto a los valores de los coeficientes $B$, todos resultaron ser significativamente positivos. Según el valor de Exp (B) cuando existió un autoconcepto familiar positivo aumentó la probabilidad de tener un PWI positivo en 119\%, el autoconcepto social aumenta en $42 \%$ y el IBL en $396 \%$. Es decir, que, para tener más probabilidades de pertenecer al grupo de jóvenes con bienestar personal, es aconsejable aumentar el bienestar local, el autoconcepto familiar y el autoconcepto social. Destacando el bienestar local como la mayor probabilidad de pertenencia al grupo de bienestar personal (tabla 6).

Las variables predictoras que conformaron el modelo, clasificaron de manera global al 79.1\% de los estudiantes en los grupos de bienestar personal y sin bienestar personal. Destacando que los que no se clasificaron en el grupo, con bienestar personal, tuvieron un porcentaje mayor con $81.3 \%$ en comparación de los que sí tienen con $76.9 \%$. Dichos porcentajes se consideran aceptables para este tipo de análisis.

\section{Tabla 6}

Resumen del análisis de regresión logística prediciendo el indice bienestar personal.

\begin{tabular}{lcccccc}
\hline Variable & $b$ & $S E$ & $O R$ & $I C 95 \%$ & Wald & $p$ \\
\hline Autoconcepto familiar & 0.78 & 0.17 & 2.19 & {$[1.55-3.08]$} & 20.15 & .000 \\
Autoconcepto social & 0.35 & 0.17 & 1.42 & {$[1.01-1.99]$} & 4.15 & .042 \\
Índice de bienestar local & 1.60 & 0.19 & 4.96 & {$[3.41-7.20]$} & 70.62 & .000 \\
\hline
\end{tabular}

Nota: $I C=$ intervalo de confianza para el odds ratio $(O R)$

\section{Análisis de Regresión Logística para la MSLSS}

En la regresión de la MSLSS el valor de $\mathrm{r}^{2}$ de Nagelkerke fue muy similar con un valor de 0.54 , significando que el modelo tuvo una capacidad de predicción adecuada. Los valores de los coeficientes $B$ fueron significativamente positivos. Entonces, cuando aumentó el autoconcepto familiar, incrementó la probabilidad en $175 \%$ de percibir una satisfacción con la vida, el autoconcepto social un 136\%, el 
bienestar local un $86 \%$ y el apego al barrio un $81 \%$ se traduce a una mayor satisfacción con la vida. (tabla 7). El modelo logró clasificar de manera general al $81.2 \%$ de los estudiantes. El modelo también predijo, en mayor medida, a los insatisfechos, quienes tuvieron un $82.2 \%$, mientras que los satisfechos consiguieron un $80.1 \%$.

\section{Tabla 7}

Resumen del análisis de regresión logistica prediciendo la satisfacción con la vida.

\begin{tabular}{lcccccc}
\hline Variable independiente & $b$ & $S E$ & $O R$ & $I C 95 \%$ & Wald & $p$ \\
\hline Autoconcepto familiar & 1.01 & 0.19 & 2.75 & {$[1.88-4.01]$} & 27.37 & .000 \\
Autoconcepto social & 0.86 & 0.19 & 2.36 & {$[1.60-3.47]$} & 19.19 & .000 \\
Bienestar local & 0.62 & 0.19 & 1.86 & {$[1.27-2.72]$} & 10.35 & .001 \\
Apego al barrio & 0.59 & 0.20 & 1.81 & {$[1.21-2.70]$} & 8.49 & .004 \\
\hline
\end{tabular}

Nota: $I C=$ intervalo de confianza para el odds ratio $(O R)$

\section{Discusión}

Los modelos de predicción que se utilizaron para identificar factores de protección y agrupar a los jóvenes satisfechos e insatisfechos o con bienestar personal y sin bienestar personal, resultaron muy similares. Hay que recordar que todos los reactivos que conformaban las variables incluidas y excluidas en los análisis son reactivos donde se comprobó su sensibilidad y discriminación para la presente población. A pesar de que se consideraron cuatro dimensiones de autoconcepto, cinco de activos de barrio y el IBL, en los cuatro modelos fueron el IBL, el autoconcepto familiar y el autoconcepto social las variables protectoras y clasificadoras, sumándose el apego al barrio en el análisis discriminante y regresión logística para la MSLSS, quedando fuera en los modelos las variables personales de autoconcepto académico y autoconcepto físico, las variables comunitarias de apoyo y empoderamiento de la juventud, seguridad del barrio, control social y las actividades para jóvenes. 
La dimensión de apego al barrio fue la única dimensión de los activos de barrio que resultó como variable de clasificación y protectora para la satisfacción con la vida. Estos resultados concuerdan con un estudio realizado por Ríos y Moreno (2010), en el cual analizaron la satisfacción con la vida y su relación con la identidad y el apego al barrio en un grupo de inmigrantes y otro de autóctonos. En dicho estudio se concluyó que el apego al barrio predecía la satisfacción con la vida en ambos grupos de habitantes. Además, para las personas autóctonas la participación en sus comunidades no era tan importante como el sentido de pertenencia. Esto último podría explicar por qué el activo de barrio de apoyo y empoderamiento no resultó como variable predictora y el apego al barrio sí.

El autoconcepto familiar y autoconcepto social fueron variables predictoras que resultaron significativas en los cuatro modelos. Esto se puede explicar, ya que como se mencionó anteriormente, los sonorenses de comunidades marginadas tienden a ser más colectivos que la clase media y suelen dar una mayor relevancia a este tipo de variables relacionadas a las interacciones que mantienen los individuos con otros seres cercanos a ellos (Díaz-Loving et al., 2018; Vera et al., 2003; Vera, Rodríguez et al., 2018).

Las investigaciones se enfocaban en los factores externos para medir la calidad de vida, sin embargo, después de años de diferentes estudios, se ha concluido que las condiciones externas solo juegan una parte en la explicación del bienestar (Rojas, 2005). Existen aspectos universales con los cuales nunca se ha correlacionado negativamente el bienestar, como lo son los ingresos, afectos positivos o la extraversión. Sin embargo, existen variaciones en otros factores dependiendo de las culturas (Suh y Choi, 2018).

El bienestar local fue otra de las variables que resultó relevante para predecir el bienestar personal y la satisfacción con la vida, coincidiendo con lo encontrado por Tánori (2012) y Arita (2005), quienes encontraron correlación en todas las dimensiones. Y a su vez, se comprobó que la inseguridad no contribuye al bienestar subjetivo por lo cual el apego al barrio, que mide sobre todo el sistema de identidad del joven 
con su propio espacio público, podría ser un mediador entre el bienestar personal y el bienestar local.

En síntesis, el bienestar personal y la satisfacción con la vida de los adolescentes estudiantes del noroeste de México (Sonora), depende de las variables personales vinculadas a las relaciones interpersonales, así como de encontrarse conforme y apegado a la comunidad en la cual se vive. Ambos modelos permiten clasificar a más del 75\% de los casos, resultando el modelo de predicción con la MSLSS el que puede clasificar a un mayor porcentaje de población global.

Se encontró de las variables analizadas al autoconcepto social y familiar como los mayores predictores de la satisfacción con la vida en los adolescentes del noroeste de México, dichos términos se relacionan íntima e implícitamente con las relaciones interpersonales y apoyo social.

La evidencia empírica previa a este estudio muestra que la satisfacción con la vida es integral para el funcionamiento positivo y exitoso en la juventud. Las relaciones interpersonales con los amigos y con las familias, así como el apoyo de estos durante la adolescencia, representan un papel determinante en el desarrollo del bienestar personal en los adolescentes (Proctor y Linley, 2014; Raboteg-Saric y Sakic, 2014, Rodríguez et al., 2012).

Jóvenes con mayores niveles de satisfacción con la vida se benefician de una variedad de resultados positivos, incluidos el funcionamiento psicosocial adaptativo, mejores relaciones interpersonales y sociales, menos problemas de comportamiento y una serie de resultados positivos relacionados con la escuela, entre los que se incluyen un mayor éxito académico, mayor satisfacción escolar, competencia y autoeficacia. Claramente, experimentar altos niveles de satisfacción con la vida es deseable para los jóvenes, puesto que conducen a una gama de gratos resultados para la juventud (Proctor y Linley, 2014).

Como se ha comprobado en otros estudios (Rodríguez, et al., 2016; Galinha y Pais- Ribeiro, 2012), se confirmó que el peso de las variables psicológicas es mayor que la influencia que tienen los factores contextuales sobre la satisfacción con la vida. 
En resumen, los análisis discriminantes y de regresión logística han permitido constatar como atributos importantes en la predicción del bienestar personal al IBL, el autoconcepto familiar y autoconcepto social y en caso de los modelos para la MSLSS se ańade la variable del apego al barrio.

En base a regresiones logísticas se encontró que el autoconcepto familiar fue la variable que produce mayor probabilidad (175\%) de pasar de insatisfecho a satisfecho. En la regresión logística de bienestar personal el bienestar local es el que produce una mayor probabilidad (396\%) de pasar al grupo con un nivel de bienestar personal.

Los resultados se añaden a la evidencia que indica que se debe apostar por modelos explicativos de la satisfacción con la vida como los propuestos en otros estudios (Moreno y Vera, 2011), los cuales integran tanto variables de carácter intraindividual como factores ambientales y sociales. Este tipo de modelos son los que más se ajustan y explican la realidad de los adolescentes y su interacción con el contexto que los rodea (Crous et al., 2018; Sarriera et al., 2018; Casas, 2011).

Los niños y jóvenes de hoy se convertirán en los ciudadanos, trabajadores y padres del mañana. Las escuelas desempeñan un papel crucial para mejorar su salud mental, su bienestar y su capacidad de recuperación (Proctor y Linley, 2014). Por ello, se debería reflexionar sobre la relación del bienestar subjetivo con el ajuste y rendimiento escolar de los niños y adolescentes en futuros estudios. Además, se debe destacar que la satisfacción de los adolescentes depende en parte de las condiciones de la comunidad en la que vive, lo que es relevante, puesto que dichas comunidades comúnmente no se encuentran en la mejor de las condiciones para el debido desarrollo personal y social de los adolescentes, a causa de la pobre o mala inversión del estado (Azaola, 2017).

Como limitación del estudio se debe tener presente que los resultados son pertenecientes a adolescentes escolarizados en un rango de edad de entre 12 y 15 años y que asisten a escuelas secundarias en zonas de violencia. Por ello, lo más conveniente para futuras investigaciones sería tomar muestras de población adolescente no escolarizada y de características sociodemográficas más universales, para posteriormente 
poder generalizar con mayor amplitud los datos empíricos. Por otro lado, el estudio fue de tipo transversal y no experimental, esto limita a solo conocer la situación de los sujetos en un solo momento y no poder establecer relaciones causales. Es necesario analizar el fenómeno con estudios longitudinales o experimentales para análisis más profundos de las relaciones y los constructos.

Para futuros estudios se deben considerar más variables de tipo personal, social, económico o de contexto. Las variables analizadas en este estudio se seleccionaron en función a la teoría revisada, sin embargo, estas variables son positivas por lo que actúan como protectoras y limita el análisis en este sentido. Para próximas investigaciones se recomienda añadir variables que actúen como factores de riesgo para contar con un panorama más amplio del fenómeno.

\section{Referencias}

Alfaro, J., Casas, F. y López, V. (2015). Bienestar en la infancia y adolescencia. Psicoperspectivas, 14(1), 1-5.

Arita, B. (2005). Satisfacción por la vida y teoría homeostática del bienestar. Psicología y Salud, 15(1), 121-126.

Arita, B. (2010). Avances en el estudio de la calidad de vida en Sinaloa: una década de investigación. La psicología social en México, XII, 25-30.

Arita, B. (2014). Satisfacción por la vida y teoría homeostática del bienestar. Psicología y Salud, 15(1), 121-126.

Azaola, E. (2017). Factores que contribuyen a la participación de adolescentes en delitos violentos en México. Abya-Yala: Revista sobre acceso a la justicia y los derechos en las Américas, 1(3), 7-24. https://doi.org/10.26512/abya-yala.v1i3.7115

Benatuil, D. (2003). El bienestar psicológico en adolescentes desde una perspectiva cualitativa. Psicodebate, 3, 43-58. https://doi. org/10.18682/pd.v3i0.502 
Casas, F. (2011). Subjective social indicators and child and adolescent well-being. Child Indicators Research, 4(4), 555-575. http:// dx.doi.org/10.1007/s12187-010-9093-z

Casas, F., Fernández, J., Montserrat, C., Fernández- Artamendi, Bertrán, I. y Sierra, J. (2009). Personal well-being: Age differences in two Spanish samples, according to three different instruments. IX ISQOLS Conference. Florence, Italy.

Casas, F., Fernández-Artamendi, S., Montserrat, C., Bravo, A., Bertrán, I. y Valle, J. (2013). El bienestar subjetivo en la adolescencia: Estudio comparativo de dos comunidades autónomas en España. Anales de Psicología, 29(1), 148-158. http://dx.doi. org/10.6018/analesps.29.1.145281

Casas, F., Figuer, C., González, M., Malo, S., Alsinet, C. y Subarroca, S. (2007). The well-being of 12- to 16-yearold adolescents and their parents: Results from 1999 to 2003 Spanish samples. Social Indicators Research, 83(1), 87-115. https://doi.org/10.1007/ s11205-006-9059-1

Castellá, J., Saforcada, E., Tonon, G., Rodríguez de La Vega, L., Mozobancyk, S. y Bedin, L. (2012). Bienestar subjetivo de los adolescentes: un estudio comparativo entre Argentina y Brasil. Psychosocial Intervention, 21(3), 273-280. https://doi. org/10.5093/in2012a24

Coenders, G., Casas, F., Figuer, C. y González, M. (2005). Relationships between parents' and children's salient values for future and children's overall satisfaction. A comparison across countries. Social Indicators Research, 73, 141-177. https://doi.org/10.1007/ s11205-004-3233-0

Cohen, M. A. (2008). The effect of crime on life satisfaction. The Journal of Legal Studies, 37(S2), S325-S353. https://www. jstor.org/stable/10.1086/588220

Crous, G., Casas, F. y González-Carrasco, M. (2018). What Aspects are Important to Adolescents to Achieve Full Satisfaction in Life? Child Indicators Research, 1-20. https://doi.org/10.1007/ s12187-018-9535-6 
Cummins, R. (1997). Comprehensive quality of life scale, (5a ed). Australia, School of Psychology. Deakin University.

Cummins, R. (2000). Objective and subjective quality of life: An interactive model. Social Indicators Research, 52(1), 55-72. https:// doi.org/10.1023/A:1007027822521

Cummins, R. A., Eckersley, R., Pallant, J., Van Vugt, J. y Misajon, R. (2003). Developing a national index of subjective wellbeing: The Australian Unity Wellbeing Index. Social indicators research, 64(2), 159-190. https://doi.org/10.1023/A:1024704320683

Díaz-Loving, R., Cruz-Torres, C. E., Armenta-Huarte, C. y Reyes-Ruiz, N. E. (2018). Variations in horizontal and vertical individualism-collectivism in four regions of Mexico/Variaciones en el individualismo-colectivismo horizontal y vertical en cuatro regiones de México. Revista de Psicología Social, 33(2), 329-356. https://doi.org/10.1080/02134748.2018.1435217

Diener, E. (1984). Subjective Well-being. Psychological Bulletin, 95, 542-575. http://dx.doi.org/10.1037/0033-2909.95.3.542

Diener, E. (1994). Assessing subjective well-being: Progress and opportunities. Social Indicators Research, 31(2), 103-157. https://doi. org/10.1007/BF01207052

Galindez, E. y Casas, F. (2011). Adaptación y validación de la MSLSS de satisfacción vital multidimensional con una muestra de adolescentes. Revista de Psicología Social, 26(3), 309-323. https:// doi.org/10.1174/021347411797361284

Galinha, I. y Pais-Ribeiro, J. L. (2011). Cognitive, affective and contextual predictors of subjective wellbeing. International Journal of Wellbeing, 2(1), 34-53. https://doi.org/10.5502/ijw.v2i1.3

Garaigordobil, M., Aliri, J. y Fontaneda, I. (2009). Bienestar psicológico subjetivo: diferencias de sexo, relaciones con dimensiones de personalidad y variables predictoras. Psicología Conductual, 17(3), 543-559.

García, F. y Musitu, G. (1999). AF5: Autoconcepto Forma 5. TEA Ediciones. 
George, D. y Mallery, M. (2003). Using SPSS for Windows step by step: a simple guide and reference. Boston: Allyn y Bacon.

González, C., Vera, J. y Bautista, G. (2015) Bienestar subjetivo en la población indígena de México. En Gamboa, M., Medina, F., Mendoza, E., Ramírez, M., Terré, O. (Comps.), Formación docente y prácticas inclusivas (pp. 88-104). IFODES.

Helliwell, J., Huang, H. y Wang, S. (2017). Chapter 2: The social foundations of world happiness. En Helliwell, J., Layard, R. y Sachs, J.( Eds.), World happiness report 2017 (pp. 8-47). Sustainable Development Solutions Network.

Huebner, E. (2004). Research on assessment of life satisfaction of children and adolescents. Social Indicators Research, 66(1-2), 3-33. https://doi.org/10.1023/B:SOCI.0000007497.57754.e3

Huebner, E. S. (2001). Manual for the Multidimensional Students' Life Satisfaction Scale. University of South Carolina.

INEGI (2015). Indicadores de bienestar subjetivo de la población adulta en México. Boletín de prensa 412/15. México: Aguascalientes, Ags.

Malo, S., Bataller, S., Casas, F., Gras, M. y González, M. (2011). Análisis psicométrico de la escala multidimensional de autoconcepto AF5 en una muestra de adolescentes y adultos de Cataluña. Psicothema, 23(4).

Martínez, M., Buelga, S. y Cava, M. J. (2007). La satisfacción con la vida en la adolescencia y su relación con la autoestima y el ajuste escolar. Anuario de Psicología, 38(2), 5-15.

Martínez-Ferrer, B., Ávila-Guerrero, M. E., Vera-Jiménez, J. A., Bahena-Rivera, A. y Musitu-Ochoa, G. (2016). Satisfacción con la vida, victimización y percepción de inseguridad en Morelos, México. Salud Pública de México, 58(1), 16-24.

Moreno, J. A. y Vera, J. A. (2011). Modelo causal de la satisfacción con la vida en estudiantes adolescentes. Revista de Psicodidáctica, 16(2), 367-380.

Moreno-Ruiz, D., Povedano, A., Martínez, B. y Musitu, G. (2012). Emotional and Social Problems in Adolescents from a Gender 
Perspective Universidad. The Spanish Journal of Psychology, 15(3), 1013-1023. https://doi.org/10.5209/rev_SJOP.2012. v15.n3.39392

OCCSES (2016). Análisis Regional. Recuperado de: www.observatoriodesonora.org/que.html.

OECD (2013). OECD Guidelines on Measuring Well-being in Mexican States. OECD Publishing, Paris.

Oliva, A., Antolín, L., Estévez, R. M. y Pascual, D. M. (2012). Activos del barrio y ajuste adolescente. Intervención Psicosocial, 21, 17-27. http://dx.doi.org/10.5093/in2012v21n1a1

Oliva, A., Pertegal, M., Antolín, L., Reina, M., Ríos, M., Hernando, Á. y Parra, Á. (2011). Desarrollo positivo adolescente y los activos que lo promueven: un estudio en centros docentes andaluces. Sevilla: Junta de Andalucía.

Palomar, J. (2010). Resiliencia y bienestar subjetivo ¿son conceptos similares? La Psicología Social en México, XII, 863-870.

PNUD (2011). ¿Para qué medir bienestar subjetivo y psicológico? Boletín no. 80.

Proctor, C. y Linley, P. A. (2014). Life satisfaction in youth. En G. A. Fava y C. Ruini (Eds.), Increasing Psychological Well-Being in Clinical and Educational Settings: Interventions and Cultural Contexts (pp. 199-215). New York, NY: Springer Science.

Raboteg-Saric, Z. y Sakic, M. (2014). Relations of parenting styles and friendship quality to self-esteem, life satisfaction and happiness in adolescents. Applied Research in Quality of Life, 9(3), 749-765. http://dx.doi.org/10.1007/s11482-013-9268-0

Ríos, M. y Moreno, M. (2010). Influencia de la participación comunitaria y la identidad con el lugar en la satisfacción vital en inmigrantes. Escritos de Psicología (Internet), 3(2), 8-16.

Rodríguez, A., Droguett, L. y Revuelta, L. (2012). Ajuste escolar y personal en la adolescencia: El papel del autoconcepto académico y del apoyo social percibido. Revista de Psicodidáctica, 17(2). http://dx.doi.org/0.1387/Rev.Psicodidact.3002

Rodríguez, A., Ramos, E., Ros, I., Fernández, A. y Revuelta, L. (2016). Bienestar subjetivo en la adolescencia: el papel de la resiliencia, 
el autoconcepto y el apoyo social percibido. Suma Psicológica, 23(1), 60-69. http://dx.doi.org/10.1016/j.sumpsi.2016.02.002 Rojas, M. (2005). El bienestar subjetivo en México y su relación con indicadores objetivos. En L. Garduño, B. Salinas y M. Rojas (Eds.), Calidad de vida y bienestar subjetivo en México (pp. 83-111).

Sarriera, J. C., Casas, F., dos Santos, B. R., Bedin, L. M. y Gonzàlez,M. (2018). Subjective Well-Being and Personal Relationships in Childhood: Comparison of Brazilian and Spanish Children. Interpersona: An International Journal on Personal Relationships, 12(1), 91-106. https://doi.org/10.5964/ijpr.v12i1.284

Schmidt, V., de Figueroa, L. y Giménez, M. (2014). Estudio de las propiedades psicométricas de un instrumento para la evaluación de la satisfacción vital global. Calidad de Vida y Salud, 7(2), 54-67.

Shavelson R., Hubner, J. y Stanton, G. (1976). Self-concept: Validation of construct interpretation. Review of Educational research, 46(3), 407-441. https://doi.org/10.3102/00346543046003407

Suh, E. y Choi, S. (2018). Predictors of subjective well-being across cultures. En E. Diener, S. Oishi y L. Tay (Eds.), Handbook of well-being. Salt Lake City, UT: DEF Publishers.

Tánori, J. (2012). Calidad de vida, premisas históricas socio-culturales y recursos psicológicos: en tres generaciones de Sonorenses. Tesis de doctorado. Universidad Autónoma de Sinaloa. Sinaloa, México.

Tánori, J., Vera, J. y Arita, B. (2010) Bienestar personal en adolescentes hermosillenses. En S. Rivera, R. Díaz, I. Reyes, R. Sánchez y L.M. Cruz (Eds.), La psicología social en México Vol. XIII, (pp. 695-702). Editorial AMEPSO.

Veenhoven, R. (2008). Sociological theories of subjective well-being. The science of subjective well-being, 9, 44-61.

Vera J., Rodríguez C. y Grubits H. (2017). Satisfacción con la vida en personas con problemas auditivos. En Gamboa R. M., Medina F.L., Ramírez Z.M., Terré C.O., Enríquez F.M. (Comps.), Inclusión y prácticas educativas exitosas (pp. 163-170). IFODES. 
Vera, J. A., Tánori, J., Bautista, G. y Rodríguez, C. K. (2018). Bienestar subjetivo en personas con discapacidad motriz en México. Revista Psicologia e Saúde, 10(2), 15-24. http://dx.doi.org/10.20435/ pssa.v9i1.375.

Vera, J., Alvarez, R., Batista, Pimentel, C. y Vasconcelos, T. (2010) Bem estar subjetivo em estudantes universitários paraibanos. En S. Fernández, C. Pimentel, V. Gouveia y J. Estramiana (Eds.), Psicologia social: perspectivas actuais e evidências empiricas, (pp. 180-204). Sao Paulo: Casa do Psicólogo.

Vera, J., Batista, F., Laborín, J., Morales, A. y Torres, M. (2002) Autocenceito em uma populacao do Nordeste Brasileiro SCALE Validation of self-concept in Northeast Brazilians. PSICO. 33(1), 37-51.

Vera, J., Domínguez, M., Laborín, J., Batista, J., Seabra, M. (2007) Autoconcepto, Locus de Control y Orientación al Éxito: sus relaciones predictivas en adultos mayores del Noreste Brasileño. Psicología USP, 18(1), 137-151. http://dx.doi.org/10.1590/ S010365642007000100008I

Vera, J., Laborín, J., Domínguez, S. y Peña, M. (2003). Identidad psicológica y cultural de los sonorenses. Región y Sociedad, 15(28), 3-45.

Vera, J. y Rodríguez, E. (2007) La felicidad y sus correlatos en estudiantes de la Universidad de Sonora. Revista Universidad de Sonora. 19, 17-19.

Vera, J., Rodríguez, C., Tánori, J. y Grubits, H. (2018). Recursos de ajuste psicosocial y su relación con la satisfacción con la vida en jóvenes de México. Pensamiento Psicológico, 16(2), 87-97. http:// dx.doi.org/10.11144/Javerianacali.PPSI16-2.rapr

Recibido: 2019-10-01

Revisado: 2021-04-08

Aceptado: 2021-06-01 